\title{
BIOMASSA E ATIVIDADE MICROBIANA EM SOLO SOB DIFERENTES SISTEMAS DE MANEJO NA REGIÃO FISIOGRÁFICA CAMPOS DAS VERTENTES - $\mathrm{MG}^{(1)}$
}

\author{
Rubens Ribeiro da Silva ${ }^{(2)}$, Marx Leandro Naves Silva ${ }^{(3)}$, Evaldo Luis \\ Cardoso $^{(4)}$, Fátima Maria de Souza Moreira ${ }^{(3)}$, Nilton Curi ${ }^{(3)} \&$ \\ Alessandra Mayumi Tokura Alovisi ${ }^{(5)}$
}

\begin{abstract}
RESUMO
A biomassa e a atividade microbiana têm sido apontadas como indicadores adequados de alterações provocadas por diferentes sistemas de uso e manejo do solo. $\mathrm{O}$ objetivo deste trabalho foi avaliar as alterações na biomassa e na atividade microbiana de um Latossolo Vermelho-Amarelo ácrico típico, sob Cerrado nativo e diferentes sistemas de manejo, na região fisiográfica Campos das Vertentes, Minas Gerais. Os sistemas avaliados foram: cultivo convencional com batata (CCB); cultivo com batata, sucedido por aveia e rotação com milho (CBAM); cultivo convencional com milho (CCM); plantio direto com milho (PDM); cultivo convencional com eucalipto (CCE); e o Cerrado nativo (CN) como referência. Foram coletadas amostras de solo nas profundidades de 0-10, 10-20 e 20-30 cm, analisados o C microbiano (Cmic) e a respiração basal e calculados o quociente metabólico $\left(q \mathrm{CO}_{2}\right)$ e a relação $\mathrm{Cmic/C}$ orgânico. A biomassa e a atividade microbiana foram influenciadas pelos diferentes sistemas de manejo do solo, e as condições mais satisfatórias para a microbiota do solo ocorreram no Cerrado nativo. O manejo mais intensivo do solo e o uso frequente de agrotóxicos, característicos dos sistemas CCB e CBAM, determinaram redução nos teores de Cmic, menores valores da relação $\mathrm{Cmic/C}$ orgânico e maiores valores de $q \mathrm{CO}_{2}$, indicando uma provável condição de estresse para a biomassa microbiana.
\end{abstract}

Termos de indexação: carbono microbiano, qualidade do solo, matéria orgânica.

\footnotetext{
(1) Parte da Dissertação de Mestrado do primeiro autor apresentada ao Curso de Pós-Graduação em Ciência do Solo da Universidade Federal de Lavras - UFLA. Recebido para publicação em outubro de 2009 e aprovado em julho de 2010.

(2) Professor da Universidade Federal do Tocantis, Campus de Gurupi, Caixa Postal 66, CEP 77402-970 Gurupi (TO). E-mail: rrs2002@uft.edu.br

(3) Professor do Departamento de Ciência do Solo, Universidade Federal de Lavras - UFLA. Caixa Postal 37, CEP 37200-000 Lavras (MG).E-mails: marx@ufla.br; fmoreira@ufla.br, niltcuri@ufla.br

(4) Pesquisador da Embrapa Pantanal. Caixa Postal 109, CEP 79320-900 Corumbá (MS). E-mail: evaldo@cpap.embrapa.br

(5) Professora da Faculdade Anhanguera de Dourados. Rua Rio Brilhante 667, CEP 79811-120 Dourados (MS). E-mail: agronomia.dourados@unianhanguera.edu.br
} 


\title{
SUMMARY: SOIL BIOMASS AND MICROBIAL ACTIVITY UNDER DIFFERENT MANAGEMENT SYSTEMS IN THE PHYSIOGRAPHIC REGION CAMPOS DAS VERTENTES - MINAS GERAIS
}

\begin{abstract}
Biomass and microbial activity have been suggested as appropriate indicators of changes caused by different land use and management systems. The objective of this study was to evaluate alterations in biomass and microbial activity of a typic acric Red-Yellow Latosol (Oxisol), under native Cerrado and different management systems, in the physiographic region Campos das Vertentes, state of Minas Gerais. The evaluated systems were: conventional tillage with potato (CCB); potato followed by oat in rotation with corn (CBAM); conventional tillage with corn (CCM); no-till with corn (PDM); conventional tillage with eucalyptus (CCE); and native Cerrado (CN) as reference. Soil samples were collected in the layers 0-10, 10-20 and 20-30 cm. The microbial carbon (Cmic) and basal respiration were analyzed, and the metabolic quotient $\left(\mathrm{qCO}_{2}\right)$ and $\mathrm{Cmic} /$ organic carbon ratio were calculated. The biomass and microbial activity were influenced by the different soil management systems and the conditions for soil microbiota were most adequate under NC. The most intensive soil managements with frequent pesticide use, as in the CCB and CBAM systems, resulted in lower amounts of Cmic, lower Cmic/TOC ratio and higher $\mathrm{qCO}_{2}$ values, indicating a probable stress condition for microbial biomass.
\end{abstract}

Index terms: microbial carbon, soil quality, organic matter.

\section{INTRODUÇÃO}

Nas últimas décadas, a preocupação com a avaliação da qualidade do solo tem merecido destacada atenção, e a quantificação de alterações nos seus atributos, decorrentes da intensificação de sistemas de uso e manejo, tem sido amplamente realizada para monitorar a produtividade sustentável dos solos (Neves et al., 2007) e a conservação dos recursos naturais.

A manutenção da produtividade dos ecossistemas agrícolas e florestais depende, em grande parte, do processo de transformação da matéria orgânica e, por conseguinte, da biomassa microbiana (GamaRodrigues \& Gama-Rodrigues, 2008); por isso, tem sido crescente o interesse nos aspectos relacionados ao funcionamento biológico do solo sob sistemas naturais e agrícolas (Matsuoka et al., 2003). Como a atividade da microbiota do solo é responsável pela decomposição dos compostos orgânicos, pela ciclagem de nutrientes e pelo fluxo de energia do solo, a biomassa microbiana e sua atividade têm sido apontadas como as características mais sensíveis às alterações na qualidade do solo, causadas por mudanças de uso e práticas de manejo (Trannin et al., 2007).

A biomassa microbiana é o compartimento da matéria orgânica do solo diretamente influenciado por fatores bióticos e abióticos, de tal forma que respostas a mudanças nos sistemas de uso e manejo do solo podem ser detectáveis muito mais rapidamente pela biomassa microbiana e seus metabólitos do que nos teores de $\mathrm{C}$ do solo, principalmente devido ao tempo de ciclagem da matéria orgânica (Gama-Rodrigues et al., 2005). Estimativas da biomassa microbiana têm sido usadas em estudos do fluxo de $\mathrm{C}$ e N , ciclagem de nutrientes e produtividade das plantas em diferentes ecossistemas terrestres, possibilitando ainda a associação da quantidade de nutrientes imobilizados e a atividade da biomassa microbiana com a fertilidade e o potencial de produtividade do solo (Gama-Rodrigues \& Gama Rodrigues, 2008).

A quantidade e composição da biomassa microbiana podem ser influenciadas por diversos fatores, entre os quais o sistema de cultivo, a rotação de culturas e a textura do solo (Venzke Filho et al., 2008). A influência das plantas sobre a biomassa microbiana pode ser direta, como no caso do efeito seletivo da rizosfera (Neal et al., 1973), ou indireta, por meio das fontes de $\mathrm{C}$ provenientes dos resíduos culturais, que podem ser mais ou menos biodisponíveis à decomposição enzimática pelos microrganismos (Herman et al., 1977; Rahn \& Lillywhite, 2001). Por outro lado, muitas vezes o crescimento microbiano pode ser limitado pela escassez de nutrientes encontrados no solo, mas a adição de fontes de $\mathrm{C}$ ou $\mathrm{N}$ pode aumentar a biomassa e, com isso, imobilizá-los na sua constituição celular (Graham et al., 2002).

A atividade da microbiota do solo pode ser avaliada de diversas formas, como pela medição da sua biomassa, da atividade de certas enzimas no solo, medidas da respiração basal (Tótola \& Chaer, 2002), entre outras. Assim como outros processos metabólicos, a respiração é dependente do estado fisiológico da célula microbiana e é influenciada por diversos fatores do solo, como: a umidade, a temperatura, a estrutura, a disponibilidade de nutrientes, a textura, a relação $\mathrm{C} / \mathrm{N}$, a presença de resíduos orgânicos, entre outros. Altas taxas de respiração podem indicar tanto um distúrbio ecológico como um alto nível de produtividade do ecossistema 
(Islam \& Weil, 2000). Anderson \& Domsch (1993) propuseram o quociente metabólico $\left(q \mathrm{CO}_{2}\right)$, definido pela razão entre a respiração basal por unidade de biomassa microbiana do solo e por unidade de tempo, como atributo que permite a identificação de solos contendo biomassa mais eficiente na utilização de $\mathrm{C} \mathrm{e}$ energia, os quais refletem ambientes com menor grau de distúrbio ou estresse (Chaer \& Tótola, 2007). Valores mais elevados de $q \mathrm{CO}_{2}$, normalmente, são associados com ecossistemas jovens, submetidos a alguma condição de estresse, enquanto menores valores, normalmente, são associados com ecossistemas maduros e estáveis (Anderson \& Domsch, 1993).

Nesse contexto, o objetivo deste trabalho foi avaliar as alterações na biomassa e na atividade microbiana de um Latossolo Vermelho-Amarelo ácrico típico, sob Cerrado nativo e diferentes sistemas de manejo, na região Campos das Vertentes, Minas Gerais.

\section{MATERIAL E MÉTODOS}

O trabalho foi realizado na região Campos das Vertentes, localizada na bacia do Alto do Rio Grande, situada no centro-sul do Estado de Minas Gerais. O solo das áreas de estudo foi classificado como Latossolo Vermelho-Amarelo ácrico típico, textura muito argilosa (Embrapa, 1999), desenvolvido sobre um substrato geológico correspondente a rochas metapelíticas pobres dos grupos São João Del Rei (filito) e Andrelândia (micaxisto) (Quadro 1).

A descrição detalhada dos diferentes sistemas de uso e manejo do solo, com o respectivo histórico das áreas de estudo, constam no quadro 2. Em janeiro de 2001 foi realizada uma coleta de amostras de solo nas entrelinhas dos diferentes sistemas de manejo e aleatoriamente no Cerrado nativo, nas profundidades de 0-10, 10-20 e 20-30 cm do perfil do solo, compostas de três subamostras e com três repetições. As amostras foram acondicionadas em sacos plásticos, protegidas da luz e mantidas em caixas térmicas, sendo transportadas para o laboratório no prazo máximo de $48 \mathrm{~h}$. Em seguida, foram peneiradas (malha de $2 \mathrm{~mm}$ ), com a umidade do solo uniformizada para 50 a $60 \%$ da capacidade máxima de retenção de água, acondicionadas em sacos plásticos e mantidas sob refrigeração próxima a $4{ }^{\circ} \mathrm{C}$.

O C da biomassa microbiana (Cmic) foi determinado pelo método da fumigação-extração (Vance et al., 1987), que consiste na extração do Cmic, após a aplicação de clorofórmio às amostras, o que provoca morte dos microrganismos e liberação dos componentes celulares. O cálculo do teor de Cmic foi realizado a partir da seguinte expressão:

$$
\text { Cmic }=\frac{(F-N F)}{K c}
$$

em que Cmic é o C da biomassa microbiana $\left(\mathrm{mg} \mathrm{g}^{-1}\right)$; $\mathrm{F}$, o C da amostra fumigada ( $\left.\mathrm{mg} \mathrm{g}^{-1}\right) ; \mathrm{NF}$, o C da amostra não fumigada ( $\left.\mathrm{mg} \mathrm{g}^{-1}\right)$; e Kc, o fator para converter o C extraído em Cmic. Os valores de Kc citados na literatura são variáveis, não havendo consenso quanto ao valor a ser utilizado. Com base no clima e tipo de solo, adotou-se neste estudo o valor de Kc de 0,30 (Feigl et al., 1995), conforme sugerido por Moreira \& Siqueira (2006) para solos ácidos e tropicais.

A partir dos valores do Cmic e do conteúdo de C orgânico (CO), foi calculado o quociente microbiano ou relação Cmic/CO, de acordo com Sparling (1992). A atividade respiratória da biomassa microbiana, ou respiração basal do solo, foi determinada pela quantificação de $\mathrm{C}-\mathrm{CO}_{2}$ produzido a partir de $20 \mathrm{~g}$ de solo, incubados por $72 \mathrm{~h}$ em sistema fechado, sendo o C- $\mathrm{CO}_{2}$ capturado em solução de $\mathrm{NaOH} 0,05 \mathrm{~mol} \mathrm{~L}^{-1}$, que foi titulada com $\mathrm{HCl} 0,05 \mathrm{~mol} \mathrm{~L}^{-1}$ (Isermeyer, 1952). O quociente metabólico $\left(q \mathrm{CO}_{2}\right)$ foi calculado de acordo com Anderson \& Domsch (1993), pela razão entre a respiração basal e o Cmic.

Os resultados foram submetidos à análise de variância, segundo delineamento experimental inteiramente casualizado com três repetições, conforme

Quadro 1. Caracterização física e química do Latossolo Vermelho-Amarelo ácrico típico, na profundidade de 0-20 cm, sob diferentes sistemas de uso e manejo, na região fisiográfica Campos das Vertentes, MG

\begin{tabular}{|c|c|c|c|c|c|c|c|c|c|c|c|c|}
\hline Sistema & $\mathbf{p H}$ & $\mathbf{P}$ & $\mathbf{K}$ & $\mathrm{Ca}^{2+}$ & $\mathrm{Mg}^{2+}$ & $\mathbf{H}+\mathbf{A l}$ & $\mathrm{Al}^{3+}$ & $\mathbf{S}$ & $\mathbf{T}$ & V & $\mathrm{CO}$ & Argila \\
\hline & & \multicolumn{2}{|c|}{$-\mathrm{mg} \mathrm{dm} \mathrm{d}^{-3}-$} & \multicolumn{6}{|c|}{$-\mathrm{cmol}_{\mathrm{c}} \mathrm{dm}^{-3}$} & $\%$ & \multicolumn{2}{|c|}{$-\mathrm{g} \mathrm{kg}^{-1}-$} \\
\hline CCB & 5,5 & 10 & 48 & 1,6 & 0,9 & 3,6 & 0,0 & 2,6 & 6,2 & 42 & 22 & 637 \\
\hline CBAM & 6,0 & 1 & 18 & 1,5 & 0,6 & 2,5 & 0,0 & 2,1 & 4,6 & 45 & 23 & 529 \\
\hline CCM & 5,9 & 3 & 35 & 2,2 & 1,2 & 2,8 & 0,0 & 3,4 & 6,2 & 55 & 26 & 476 \\
\hline PDM & 6,0 & 3 & 53 & 2,4 & 1,4 & 2,8 & 0,0 & 3,9 & 6,7 & 59 & 29 & 525 \\
\hline $\mathrm{CCE}$ & 5,2 & 3 & 15 & 1,0 & 0,2 & 7,7 & 0,6 & 1,1 & 8,8 & 15 & 23 & 605 \\
\hline $\mathrm{CN}$ & 5,2 & 2 & 17 & 0,6 & 0,2 & 6,1 & 0,3 & 0,8 & 6,8 & 12 & 27 & 659 \\
\hline
\end{tabular}

CCB: cultivo convencional com batata; CBAM: cultivo convencional com batata, sucedido por aveia e rotacionado com milho; CCM: cultivo convencional com milho; PDM: plantio direto com milho; CCE: cultivo convencional com eucalipto; CN: Cerrado nativo. S: soma de bases; T: capacidade de troca catiônica a pH 7.0; V: saturação por bases; CO: C orgânico. 
Quadro 2. Histórico e descrição das áreas experimentais (tratamentos) no Latossolo Vermelho-Amarelo ácrico típico, na região Campos das Vertentes, bacia do Alto do Rio Grande, MG

\begin{tabular}{|c|c|}
\hline Símbolo & Histórico e descrição da área \\
\hline $\mathrm{CCB}$ & $\begin{array}{l}\text { - cultivo convencional com batata por um ano (ano agrícola 2000/2001). O preparo do solo consistiu de } \\
\text { uma aração e uma gradagem niveladora, e o plantio foi realizado manualmente em sulcos. A } \\
\text { "amontoa" foi realizada aproximadamente } 16 \text { dias após a emergência e acompanhada da aplicação de } \\
\text { inseticida (carbofuran), na dose de } 30 \mathrm{~kg} \mathrm{ha}^{-1} \text {. O controle fitossanitário foi realizado por pulverizações } \\
\text { preventivas semanais, com fungicida (ditiocarbamato), na dose de } 3 \mathrm{~kg} \mathrm{ha}^{-1} \text {, e inseticida } \\
\text { (metamidophos), na dose de } 0,5 \mathrm{~L} \mathrm{ha}^{-1} \text {. }\end{array}$ \\
\hline CBAM & $\begin{array}{l}\text { - cultivo com batata por dois anos (1999/2000 e 2000/2001), sucedido por aveia após uma subsolagem } \\
\text { pós-colheita da batata em 1999/2000, e rotacionado com milho no plantio direto em 2000/2001. No } \\
\text { cultivo da batata foi adotado o mesmo manejo descrito no CCB. }\end{array}$ \\
\hline $\mathrm{CCM}$ & $\begin{array}{l}\text { - cultivo convencional com milho por dois anos (1999/2000 e 2000/2001), rotacionado com feijão em } \\
1999 / 2000 .\end{array}$ \\
\hline PDM & $\begin{array}{l}\text { - plantio direto com milho por cinco anos (1996/2001), conduzido após o cultivo convencional com } \\
\text { batata em 1986/1987, arroz em 1987/1989 e milho convencional em 1988/1996. }\end{array}$ \\
\hline $\mathrm{CCE}$ & $\begin{array}{l}\text { - cultivo convencional com eucalipto por } 13 \text { anos (desde 1978), no espaçamento de } 3 \text { x } 3,5 \mathrm{~m} \text {, sem } \\
\text { nenhuma prática de manejo do solo após o plantio do eucalipto. }\end{array}$ \\
\hline $\mathrm{CN}$ & $\begin{array}{l}\text { - área de Cerrado, cuja vegetação primária remanescente é representada pelo Cerrado tropical } \\
\text { subcaducifólio e campo Cerrado (Embrapa, 1988). As espécies predominantes são: Stryphnodendron } \\
\text { barbatimao (barbatimão), Cariocar brasiliense (pequizeiro), Duguetia furfuracea (araticum-pedra), } \\
\text { Jacaranda paucifolia (caroba-do-campo), Dimorphandra mollis (faveiro), Tecoma sp. (ipê-do-cerrado), } \\
\text { Hymenaea stgonocarpa hayne (jatobá-do-cerrado), Kielmeyera coriacea (pau-santo), Pouteria torta } \\
\text { (figueira-do-campo) e Echnolaena inflexa (capim-flechinha) (Minas Gerais, 1980). }\end{array}$ \\
\hline
\end{tabular}

os procedimentos do SAS (SAS, 2002). As comparações múltiplas de médias foram realizadas pelo teste de Scott-Knott (Scott \& Knott, 1974) a 5 \%, utilizandose o SISVAR (Ferreira, 2000).

\section{RESULTADOS E DISCUSSÃO}

Os diferentes sistemas de uso e manejo do solo promoveram alterações no $\mathrm{C}$ da biomassa microbiana (Cmic) e na relação $\mathrm{Cmic/C}$ orgânico (Cmic/CO), em todas as profundidades amostradas (Quadro 3). O maior teor de $\mathrm{Cmic}$ foi detectado no Cerrado nativo $(\mathrm{CN})$, notadamente na profundidade de $0-10 \mathrm{~cm}$. Isso indica uma condição mais favorável à microbiota do solo, atribuída, possivelmente, ao maior aporte contínuo e variado de substratos orgânicos provenientes da maior diversidade de espécies na vegetação nativa e com diferentes graus de suscetibilidade à decomposição, corroborando D'Andréa et al. (2002) e Cardoso et al. (2009). De acordo com Perez et al. (2004), nas condições de mata nativa, a deposição de resíduos orgânicos, a grande quantidade de raízes e a maior quantidade de água retida no solo estimulam a manutenção da microbiota do solo, enquanto solos submetidos à atividade agrícola costumam apresentar condições adversas, que, normalmente, determinam decréscimo da população
Quadro 3. Carbono microbiano (Cmic) e relação Cmic/C orgânico (Cmic/CO) em Latossolo Vermelho-Amarelo ácrico típico, sob diferentes sistemas de uso e manejo, na região fisiográfica Campos das Vertentes-MG

\begin{tabular}{lccr}
\hline \multirow{4}{*}{ Sistema } & \multicolumn{3}{c}{ Profundidade (cm) } \\
\cline { 2 - 4 } & $\mathbf{0 - 1 0}$ & $\mathbf{1 0 - 2 0}$ & $\mathbf{2 0 - 3 0}$ \\
\hline & & Cmic $(\mu \mathrm{g} \mathrm{g}-1)$ \\
CCB & $119,1 \mathrm{aC}$ & $76,4 \mathrm{aB}$ & $60,9 \mathrm{aB}$ \\
CBAM & $158,2 \mathrm{aC}$ & $102,1 \mathrm{aB}$ & $159,6 \mathrm{aB}$ \\
CCM & $325,1 \mathrm{aB}$ & $511,3 \mathrm{aA}$ & $433,7 \mathrm{aA}$ \\
PDM & $343,6 \mathrm{aB}$ & $218,6 \mathrm{aB}$ & $215,7 \mathrm{aB}$ \\
CCE & $381,3 \mathrm{aB}$ & $335,4 \mathrm{aA}$ & $260,6 \mathrm{aA}$ \\
CN & $966,5 \mathrm{aA}$ & $419,0 \mathrm{bA}$ & $306,9 \mathrm{bA}$ \\
& & Cmic/CO $(\%)$ & \\
CCB & $0,50 \mathrm{aC}$ & $0,38 \mathrm{aC}$ & $0,38 \mathrm{aD}$ \\
CBAM & $0,62 \mathrm{aC}$ & $0,52 \mathrm{aC}$ & $0,94 \mathrm{aC}$ \\
CCM & $1,14 \mathrm{bB}$ & $2,24 \mathrm{aA}$ & $2,69 \mathrm{aA}$ \\
PDM & $1,20 \mathrm{aB}$ & $0,82 \mathrm{aC}$ & $1,01 \mathrm{aC}$ \\
CCE & $1,61 \mathrm{aB}$ & $1,50 \mathrm{aB}$ & $1,54 \mathrm{aB}$ \\
CN & $2,92 \mathrm{aA}$ & $1,99 \mathrm{bA}$ & $1,82 \mathrm{bB}$ \\
\hline
\end{tabular}

CCB: cultivo convencional com batata; CBAM: cultivo convencional com batata, sucedido por aveia e rotacionado com milho; CCM: cultivo convencional com milho; PDM: plantio direto com milho; CCE: cultivo convencional com eucalipto; CN: Cerrado nativo. Médias seguidas pela mesma letra, maiúscula na coluna e minúscula na linha, não diferem entre si pelo teste de Scott-Knott a $5 \%$. 
microbiana. Matsuoka et al. (2003) ressaltam ainda que condições mais favoráveis para a biomassa microbiana em solos sob vegetação nativa podem ser atribuídas ao acúmulo de serapilheira, que condiciona menor variação e níveis mais adequados de temperatura e umidade.

As reduções mais expressivas nos teores de Cmic nos diferentes sistemas de manejo do solo, em relação ao $\mathrm{CN}$, foram observadas na profundidade de $0-10 \mathrm{~cm}$, onde são maiores a presença e a atividade da microbiota do solo, correspondendo, aproximadamente, a $88 \%$ no cultivo convencional com batata (CCB), $84 \%$ no cultivo com batata sucedido por aveia e rotacionado com milho (CBAM), $66 \%$ no cultivo convencional com milho (CCM), $64 \%$ no plantio direto com milho (PDM) e $61 \%$ no cultivo convencional com eucalipto (CCE), evidenciando que a capacidade de imobilização de C pela biomassa microbiana foi inversamente proporcional à intensidade de manejo do solo. De acordo com Bayer \& Mielniczuk (2008), sob vegetação natural o conteúdo de matéria orgânica do solo é mais estável, e com o uso agrícola esse conteúdo é, de modo geral, acentuadamente reduzido quando se utilizam métodos de preparo com intenso revolvimento do solo e sistemas de cultura com baixa adição de resíduos vegetais, condições estas que influenciam a atividade e a biomassa microbiana (Venzke Filho et al., 2008).

Além dos efeitos associados à remoção da vegetação nativa e intensidade de manejo do solo, as reduções no Cmic, possivelmente, também refletiram alterações associadas à quantidade e à qualidade dos resíduos vegetais disponíveis à microbiota (Gama-Rodrigues et al., 2008; Silva et al., 2009). Isso ficou mais evidente principalmente no sistema CCE, que, apesar de ausência de manejo do solo após o plantio do eucalipto, ainda apresentou Cmic significativamente inferior ao do $\mathrm{CN}$, notadamente na profundidade de $0-10 \mathrm{~cm}$ (Quadro 3).

Nos sistemas de cultivo de batata, o emprego de produtos químicos é prática considerada obrigatória na defesa fitossanitária (Novo \& Miranda Filho, 2006) e pode ter determinado os menores teores de Cmic nos sistemas CCB e CBAM. Esses baixos teores de Cmic nesses sistemas podem ser atribuídos tanto ao intensivo manejo do solo, característico do cultivo de batata, como também ao uso frequente de agrotóxicos. De acordo com Maluche-Baretta et al. (2007), quanto maior a perturbação efetuada numa área - como as causadas por práticas de controle de pragas e doenças-, maior tende a ser a variação na biomassa microbiana. As perturbações aqui detectadas corroboram resultados de Spain \& van Veld (1983) e Bromilow et al. (1996), os quais mostraram que alguns inseticidas aplicados ao solo promovem redução do tamanho da biomassa microbiana, bem como alterações na composição de comunidades microbianas do solo.

Embora os restos culturais dos diferentes sistemas de manejo do solo não tenham sido quantificados, é possível afirmar, com base nas características dos próprios sistemas, que a menor quantidade de palhada, sobretudo no sistema CCB, também pode ter contribuído para os menores teores de Cmic, seja por proporcionar menor cobertura do solo, e consequentemente favorecer maiores variações de temperatura e umidade, seja por disponibilizar menor quantidade de substrato orgânico para decomposição e mineralização, fatores esses que exercem destacada influência na atividade e na biomassa microbiana (Moreira \& Siqueira, 2006).

A relação $\mathrm{Cmic} / \mathrm{CO}$, que expressa quanto do $\mathrm{C}$ orgânico do solo está imobilizado na biomassa microbiana, apresentou valores que obedeceram à seguinte ordem: $\mathrm{CN}>\mathrm{CCE}>\mathrm{PDM}>\mathrm{CCM}>\mathrm{CBAM}>$ $\mathrm{CCB}$, notadamente na profundidade de $0-10 \mathrm{~cm}$ (Quadro 3). Essa observação evidencia a eficiência dos microrganismos na imobilização do carbono, que apresentou tendência inversamente proporcional à intensidade de manejo do solo. Baixos valores da relação $\mathrm{Cmic/CO}$ podem ser ocasionados por circunstâncias em que a microbiota se encontra sob algum fator de estresse (Wardle, 1994) ou devido à baixa qualidade nutricional da matéria orgânica, fazendo com que a biomassa microbiana torne-se incapaz de utilizar totalmente o C orgânico (GamaRodrigues \& Gama-Rodrigues, 2008). Portanto, os menores valores da relação $\mathrm{Cmic} / \mathrm{CO}$, principalmente nos sistemas CCB e CBAM, sugerem tanto uma provável condição de estresse para a microbiota do solo, possivelmente determinada pelo manejo intensivo do solo e pelo frequente uso de agrotóxicos, como uma baixa disponibilidade ou qualidade do substrato orgânico.

De acordo com Colozzi Filho et al. (1999), sugerese que os valores da relação $\mathrm{Cmic/CO}$ que expressam uma condição de equilíbrio do $\mathrm{C}$ no solo seriam de 2,3 para monoculturas e 4,4 para rotação de culturas (Anderson \& Domsch, 1989). Assim, em solos nos quais esses valores são maiores ou menores estaria ocorrendo, respectivamente, acúmulo ou perdas de C. Sparling (1992) sugere que a relação Cmic/CO pode ser um indicador sensível de mudanças na dinâmica da matéria orgânica de solos submetidos a diferentes práticas de manejo; contudo, essa relação é influenciada por diversos fatores, como manejo do solo e grau de estabilização do C orgânico, e, para expressar uma condição de equilíbrio ou fase de degradação de $\mathrm{C}$, seria necessário estabelecer um valor-base para situações específicas.

A atividade metabólica da biomassa microbiana, expressa pela respiração basal e pelo quociente metabólico $\left(q \mathrm{CO}_{2}\right)$, variou nos diferentes sistemas de uso e manejo do solo (Quadro 4). A maior liberação de C- $\mathrm{CO}_{2}$, notadamente na profundidade de $0-10 \mathrm{~cm}$, foi observada no $\mathrm{CN}\left(275,7 \mu \mathrm{g} \mathrm{g}^{-1} \mathrm{~h}^{-1} \mathrm{C}-\mathrm{CO}_{2}\right)$, indicando maior atividade da microbiota, possivelmente estimulada pela constante deposição de substratos orgânicos e grande quantidade de raízes. Por sua vez, os menores valores de respiração basal ocorreram no 
$\operatorname{CCM}\left(104,3 \mu \mathrm{g} \mathrm{g}^{-1} \mathrm{~h}^{-1} \mathrm{C}-\mathrm{CO}_{2}\right)$ e CCE $\left(114,4 \mu \mathrm{g} \mathrm{g}^{-1} \mathrm{~h}^{-1}\right.$ $\mathrm{C}-\mathrm{CO}_{2}$ ), não sendo constatada diferença significativa entre os demais sistemas. A esperada tendência de maior atividade respiratória da biomassa microbiana nos sistemas com menor intensidade de manejo do solo, conforme reportado em outros trabalhos (Vargas \& Scholles, 2000; Costa et al., 2006; Hungria et al., 2009; Meriles et al., 2009; Frazão et al., 2010), não foi comprovada no presente estudo. O sistema de plantio direto com milho (PDM), notadamente na profundidade de $0-10 \mathrm{~cm}$, apresentou menor produção de $\mathrm{C}-\mathrm{CO}_{2}$ que os sistemas CCB e CBAM, nos quais o manejo do solo foi mais intensivo. Isso sugere que a respiração basal não foi um bom indicador para refletir as alterações na atividade da microbiota nos diferentes sistemas de manejo avaliados no presente estudo. A ausência de diferença significativa na atividade da biomassa microbiana, quantificada pela respiração basal, em solos sob sistemas de manejo convencional e plantio direto tem sido reportada em outros estudos (D'Andréa et al., 2002; Garcia et al., 2004; Mercante et al., 2008) e, em geral, é atribuída à recente implantação dos sistemas de plantio direto avaliados.

Quanto ao quociente metabólico $\left(q \mathrm{CO}_{2}\right)$, foi constatada variação significativa somente em relação aos sistemas de uso e manejo, não havendo interação significativa entre sistemas e profundidade do solo (Quadro 4). Diferentemente da respiração basal, os resultados do $q \mathrm{CO}_{2}$, relação que expressa a quantidade de $\mathrm{C}-\mathrm{CO}_{2}$ produzido por unidade de $\mathrm{Cmic}$ e por unidade de tempo, mostraram que os sistemas CCB e CBAM, caracterizados por manejo intensivo do solo e uso frequente de agrotóxicos, apresentaram biomassa microbiana menos eficiente na utilização dos compostos orgânicos, liberando mais $\mathrm{C}$ na forma de $\mathrm{CO}_{2}$ e incorporando menos $\mathrm{C}$ aos tecidos microbianos, independentemente da profundidade do solo. A menor eficiência da biomassa microbiana nos sistemas CCB e CBAM está provavelmente associada a alguma condição de estresse, pois, como evidenciado pela relação $\mathrm{Cmic/CO}$, nessas circunstâncias a capacidade de utilização do C é diminuída. De acordo com Tótola $\&$ Chaer (2002), baixos valores de $q \mathrm{CO}_{2}$ refletem ambiente mais estável ou mais próximo do seu estado de equilíbrio e, ao contrário, valores elevados são indicativos de ecossistemas submetidos a alguma condição de estresse ou de distúrbio.

\section{CONCLUSÕES}

1. Os diferentes sistemas de manejo do solo influenciaram a biomassa e atividade microbiana, e as condições mais favoráveis para a microbiota do solo ocorreram no Cerrado nativo.

2. Os teores de $\mathrm{C}$ da biomassa microbiana (Cmic) e os valores da relação $\mathrm{Cmic/C}$ orgânico foram inversamente proporcionais à intensidade de manejo do solo.
Quadro 4. Respiração basal e quociente metabólico $\left(q \mathrm{CO}_{2}\right)$ em Latossolo Vermelho-Amarelo ácrico típico, sob diferentes sistemas de uso e manejo, na região fisiográfica Campos das Vertentes-MG

\begin{tabular}{|c|c|c|c|c|}
\hline \multirow{2}{*}{ Sistema } & \multicolumn{3}{|c|}{ Profundidade (cm) } & \\
\hline & 0-10 & $10-20$ & $20-30$ & \\
\hline & \multicolumn{4}{|c|}{ Respiração basal ( $\mu \mathrm{g} \mathrm{C}-\mathrm{CO}_{2} \mathrm{~g}^{-1}$ solo $\left.\mathrm{h}^{-1}\right)$} \\
\hline $\mathrm{CCB}$ & $188,5 \mathrm{aB}$ & $155,0 \mathrm{Aa}$ & $162,7 \mathrm{aB}$ & \\
\hline CBAM & $193,1 \mathrm{aB}$ & $131,8 \mathrm{bA}$ & $143,7 \mathrm{bB}$ & \\
\hline CCM & $104,3 \mathrm{bC}$ & $80,9 \mathrm{bB}$ & $209,8 \mathrm{aA}$ & \\
\hline PDM & $186,6 \mathrm{aB}$ & $164,1 \mathrm{aA}$ & $133,8 \mathrm{aB}$ & \\
\hline $\mathrm{CCE}$ & $114,4 \mathrm{bC}$ & $183,2 \mathrm{aA}$ & $205,3 \mathrm{aA}$ & \\
\hline \multirow[t]{2}{*}{$\mathrm{CN}$} & 275,7 aA & $168,7 \mathrm{bA}$ & $154,7 \mathrm{bB}$ & \\
\hline & \multicolumn{3}{|c|}{$q \mathrm{CO}_{2}\left(\mu \mathrm{g} \mathrm{C}-\mathrm{CO}_{2} \mu \mathrm{g} \mathrm{Cmic}{ }^{-1} \mathrm{dia}^{-1}\right)$} & Média \\
\hline $\mathrm{CCB}$ & 1,67 & 2,23 & 2,97 & $2,29 \mathrm{~A}$ \\
\hline CBAM & 1,31 & 1,53 & 0,95 & $1,26 \mathrm{~B}$ \\
\hline CCM & 0,33 & 0,16 & 0,51 & $0,33 \mathrm{C}$ \\
\hline PDM & 0,54 & 0,79 & 0,76 & $0,69 \mathrm{C}$ \\
\hline $\mathrm{CCE}$ & 0,31 & 0,55 & 0,80 & $0,55 \mathrm{C}$ \\
\hline $\mathrm{CN}$ & 0,31 & 0,41 & 0,54 & $0,42 \mathrm{C}$ \\
\hline Média & $0,74 \mathrm{a}$ & $0,94 \mathrm{a}$ & $1,09 \mathrm{a}$ & \\
\hline
\end{tabular}

CCB: cultivo convencional com batata; CBAM: cultivo convencional com batata, sucedido por aveia e rotacionado com milho; CCM: cultivo convencional com milho; PDM: plantio direto com milho; CCE: cultivo convencional com eucalipto; CN: Cerrado nativo. Médias seguidas pela mesma letra, maiúscula na coluna e minúscula na linha, não diferem entre si pelo teste de Scott-Knott a $5 \%$.

3. A respiração basal, embora tenha evidenciado maior atividade da biomassa microbiana no Cerrado nativo, não foi um indicador eficiente para refletir o efeito dos diferentes sistemas de manejo do solo.

4. O quociente metabólico $\left(q \mathrm{CO}_{2}\right)$ indicou que a biomassa microbiana foi menos eficiente na utilização dos compostos orgânicos, liberando mais $\mathrm{C}$ na forma de $\mathrm{CO}_{2}$ e incorporando menos $\mathrm{C}$ aos tecidos microbianos, nos sistemas caracterizados por manejo mais intensivo do solo.

\section{LITERATURA CITADA}

ANDERSON, T.H. \& DOMSCH, K.H. The metabolic quocient for $\mathrm{CO}_{2}\left(\mathrm{qCO}_{2}\right)$ as a specific activity parameter to assess the effects of environmental conditions, such as $\mathrm{pH}$, on the microbial biomass of forest soils. Soil Biol. Biochem., 25:393-395, 1993

ANDERSON, T.H. \& DOMSCH, K.H. Rations of microbial biomass carbon to total organic in arable soils. Soil Biol. Biochem., 21:474-479, 1989.

BAYER, C. \& MIELNICZUK, J. Dinâmica e função da matéria orgânica. In: SANTOS, G.A.; SILVA, L.S.; CANELLAS, L.P. \& CAMARGO, F.A.O., eds. Fundamentos da matéria orgânica do solo ecossistemas tropicais e subtropicais. 2.ed. Porto Alegre, Metrópole, 2008. p.7-18. 
BROMILOW, R.H.; EVANS, A.A.; NICHOLLS, P.H.; TODD, A.D. \& BRIGGS, G.G. The effects on soil fertility of repeated applications of pesticides over 20 years. Pestic. Sci., 48:63-72, 1996.

CARDOSO, E.L.; SILVA, M.L.N.; MOREIRA, F.M.S. \& CURI, N. Atributos biológicos indicadores da qualidade do solo em pastagem cultivada e nativa no Pantanal. Pesq. Agropec. Bras., 44:631-637, 2009.

CHAER, G.M. \& TÓTOLA, M.R. Impacto do manejo de resíduos orgânicos durante a reforma de plantios de eucalipto sobre indicadores de qualidade do solo. R. Bras. Ci. Solo, 31:13811396, 2007

COLOZZI FILHO, A.; BALOTA, E.L. \& ANDRADE, D.S Microrganismos e processos biológicos no sistema plantio direto. In: SIQUEIRA, J.O.; MOREIRA, F.M.S.; LOPES, A.S.; GUILHERME, L.R.G.; FAQUIN, V.; FURTINI NETO, A.E. \& CARVALHO, J.G., eds. Inter-relação fertilidade, biologia do solo e nutrição de plantas. Soil fertility, soil biology, and plant nutrition interrelationships. Viçosa, MG, SBCS; Lavras, UFLA/DCS, 1999. p.487-508.

COSTA, E.A.C.; GOEDERT, W.J. \& SOUSA, D.M.G. Qualidade de solo submetido a sistemas de cultivo com preparo convencional e plantio direto. Pesq. Agropec. Bras., 41:1185-1191, 2006.

D'ANDRÉA, A.F.; SILVA, M.L.N.; CURI, N.; SIQUEIRA, J.O. \& CARNEIRO, M.A.C. Atributos biológicos indicadores da qualidade do solo em sistemas de manejo na região do Cerrado no sul do Estado de Goiás. R. Bras. Ci. Solo, 26:913-923, 2002.

EMPRESA BRASILEIRA DE PESQUISA AGROPECUÁRIA EMBRAPA. Centro Nacional de Pesquisa de Solos. Sistema brasileiro de classificação de solos. Rio de Janeiro, 1999. $412 \mathrm{p}$

FEIGL, B.J.; SPARLING, G.P.; ROSS, D.J. \& CERRI, C.C. Soil microbial biomass in Amazonian soils: Evaluation of methods and estimates of pool sizes. Soil Biol. Biochem., 27:1467-1472, 1995

FERREIRA, D.F. Análises estatísticas por meio do SISVAR (Sistema para análise de variância) para Windows versão 4.0. In: REUNIÃO ANUAL DA REGIÃO DA BRASILEIRA DA SOCIEDADE INTERNACIONAL DE BIOMETRIA, 45., São Carlos, 2000. Anais. São Carlos, Universidade Federal de São Carlos, 2000. p.255-258.

FRAZÃO, L.A.; PICCOLO, M.C.; FEIGL, B.J.; CERRI, C.C. \& CERRI, C.E.P. Inorganic nitrogen, microbial biomass and microbial activity of a sandy Brazilian Cerrado soil under different land uses. Agric. Ecosys. Environ., 135:161-167, 2010 .

GAMA-RODRIGUES, E.F.; BARROS, N.F.; GAMARODRIGUES, A.C. \& SANTOS, G.A. Nitrogênio, carbono e atividade da biomassa microbiana do solo em plantações de eucalipto. R. Bras. Ci. Solo, 9:893-901, 2005.

GAMA-RODRIGUES, E.F.; BARROS, N.F.; VIANA, A.P. \& SANTOS, G.A. Alterações na biomassa e na atividade microbiana da serapilheira e do solo, em decorrência da substituição de cobertura florestal nativa por plantações de eucalipto, em diferentes sítios da região Sudeste do Brasil. R. Bras. Ci. Solo, 32:1489-1499, 2008.
GAMA-RODRIGUES, E.F. \& GAMA-RODRIGUES, A.C. Biomassa microbiana e ciclagem de nutrientes. In: SANTOS, G.A.; SILVA, L.S.; CANELLAS, L.P. \& CAMARGO, F.A.O., eds. Fundamentos da matéria orgânica do solo ecossistemas tropicais e subtropicais. 2.ed. Porto Alegre, Metrópole, 2008. p.159-170.

GARCIA, M.H.; MELLO, L.M.M. \& CASSIOLATO, A.M.R. Variáveis microbiológicas e produtividade do feijoeiro sob diferentes manejos do solo e calagem. Pesq. Agropec. Bras., 39:1021-1026, 2004.

GRAHAM, M.H.; HAYNES, R.J. \& MEYER, J.H. Soil organic matter content and quality: effects of fertilizer applications, burning and trash retention on a long-term sugarcane experiment in South Africa. Soil Biol. Biochem., 34:93-102, 2002.

HERMAN, W.A.; McGILL, W.B. \& DORMAAR, J.F. Effects of initial chemical composition on decomposition of roots of three grass species. J. Soil Sci., 57:205-215, 1977.

HUNGRIA, M.; FRANCHINI, J.C.; BRANDÃO JÚNIOR, O.; KASCHUK, G. \& SOUZA, R.A. Soil microbial activity and crop sustainability in a long-term experiment with three soil-tillage and two crop-rotation systems. Appl. Soil Ecol., 42:288-296, 2009.

ISERMEYER, H. Eine einfache methode zur bestimmung der bodenatmung und der karbonate im boden. Z Pflanzenernäh Bodenk, 56:26-38, 1952.

ISLAM, K.R. \& WEIL, R.R. Land use effects on soil quality in a tropical forest ecosystem of Bangladesh. Agric. Ecosys. Environ., 79:9-16, 2000.

MALUCHE-BARETTA, C.R.D.; KLAUBERG-FILHO, O.; AMARANTE, C.V.T.; RIBEIRO, G.M. \& ALMEIDA, D. Atributos microbianos e químicos do solo em sistemas de produção convencional e orgânico de maçãs no Estado de Santa Catarina. R. Bras. Ci. Solo, 31:655-665, 2007.

MATSUOKA, M.; MENDES, L.C. \& LOUREIRO, M.F. Biomassa microbiana e atividade enzimática em solos sob vegetação nativa e sistemas agrícolas anuais e perenes na região de Primavera do Leste (MT). R. Bras. Ci. Solo, 7:425-433, 2003.

MERCANTE, F.M.; SILVA, R.F.; FRANCELINO, C.S.F.; CAVALHEIRO, J.C.T. \& OTSUBO, A.A. Biomassa microbiana, em um Argissolo Vermelho, em diferentes coberturas vegetais, em área cultivada com mandioca. Acta Sci. Agron., 5:479-485, 2008.

MERILES, J.M.; VARGAS GIL, S.; CONFORTO, C.; FIGONI, G.; LOVERA, E.; MARCH, G.J. \& GUZMA'N, C.A. Soil microbial communities under different soybean cropping systems: Characterization of microbial population dynamics, soil microbial activity, microbial biomass, and fatty acid profiles. Soil Till. Res., 103:271-281, 2009.

MINAS GERAIS. Secretaria de Estado da Agricultura. Zoneamento agroclimático do Estado de Minas Gerais. Belo Horizonte, 1980. 114 p. Atlas.

MOREIRA, F.M.S. \& SIQUEIRA, J.O. Microbiologia e bioquímica do solo. 2.ed. Lavras, Universidade Federal de Lavras, 2006. 729p.

NEAL, J.L.; LARSON, R.I. \& ATKINSON, T.G. Changes in rhizosphere populations of selected physiological groups of bacteria related to substitution of specific pairs of chromosomes in spring wheat. Plant Soil, 39:209-212, 1973. 
NEVES, C.M.N.N.; SILVA, M.L.N.; CURI, N.; CARDOSO, E.L.; MACEDO, R.L.G.; FERREIRA, M.M. \& SOUZA, F.S. Atributos indicadores da qualidade do solo em sistema agrossilvopastoril no noroeste do Estado de Minas Gerais. Sci. For., 74:45-53, 2007.

NOVO, M.C.S.S. \& MIRANDA FILHO, H.S. Tuberização de dois cultivares de batata sob aplicação de sulfoniluréias. Planta Daninha, 24:115-121, 2006.

PEREZ, K.S.; RAMOS, M.L.G. \& McMANUS, C. Carbono da biomassa microbiana em solo cultivado com soja sob diferentes sistemas de manejo nos Cerrados. Pesq. Agropec. Bras., 39:567-573, 2004.

RAHN, C.R. \& LILLYWHITE, R.D. A study of the quality factors affecting the short - term decomposition of field vegetable residues. J. Sci. Food Agric., 82:19-26, 2001.

SAS Institute. SAS for linear models. Cary, 2002. 256p.

SCOTT, A. J. \& KNOTT, M. Accouter analysis methods for grouping means in the analysis of variants. Biometrics, 30:507-512, 1974.

SILVA, L.G.; MENDES, I.C.; REIS JÚNIOR, F.; FERNANDES, M.F.; MELO, J.T. \& KATO, E. Atributos físicos, químicos e biológicos de um Latossolo de Cerrado em plantio de espécies florestais. Pesq. Agropec. Bras., 44:613-620, 2009.

SPAIN, J.C. \& van VELD, P.A. Adaptation of natural microbial communities to degradation of xenobiotic compounds: Effects of concentration, exposure time, inoculum, and chemical structure. Appl. Environ. Microbiol., 45:428-535, 1983.
SPARLING, G.P. Ratio of microbial biomass carbon to soil organic carbon as a sensitive indicator of changes in soil organic matter. Austr. J. Soil Res., 30:195-207, 1992.

TÓTOLA, M.R. \& CHAER, G.M. Microrganismos e processos microbiológicos como indicadores da qualidade do solo. In: ALVAREZ V., V.H.; SCHAEFER, C.E.G.R.; BARROS, N.F.; MELLO, J.W.V. \& COSTA, L.M., eds. Tópicos em ciência do solo. Viçosa, MG, Sociedade Brasileira de Ciência do Solo, 2002. p.195-276.

TRANNIN, I.C.B.; SIQUEIRA, J.O. \& MOREIRA, F.M.S. Características biológicas do solo indicadoras de qualidade após dois anos de aplicação de biossólido industrial e cultivo de milho. R. Bras. Ci. Solo, 31:1173-1184, 2007.

VANCE, E.D.; BROOKES, P.C. \& JENKINSON, D.S. An extraction method for measuring soil microbial biomass C. Soil Biol. Biochem., 19:703-707, 1987.

VARGAS, L.K. \& SCHOLLES, D. Biomassa microbiana e produção de $\mathrm{C}-\mathrm{CO}_{2}$ e $\mathrm{N}$ mineral de um Podzólico Vermelho-Escuro submetido a diferentes sistemas de manejo. R.Bras. Ci. Solo, 24:35-42, 2000.

VENZKE FILHO, S.P.; FEIGL, B.J.; PICCOLO, M.C.; SIQUEIRA NETO, M. \& CERRI, C.C. Biomassa microbiana do solo em sistema de plantio direto na região de Campos Gerais - Tibagi, PR. R. Bras. Ci. Solo, 32:599610, 2008.

WARDLE, D.A. Metodologia para quantificação da biomassa microbiana do solo. In: HUNGRIA, M. \& ARAÚJO, R.S., eds. Manual de métodos empregados em estudos de microbiologia agrícola. Brasília, Empresa Brasileira de Pesquisa Agropecuária, 1994. p.419-436. 